POS PROCEEDINGS

\title{
The differential cross section in the dp-elastic scattering at the energies from 500 to $1000 \mathrm{MeV} / \mathrm{n}$
}

\author{
A.A Terekhin ${ }^{* 1}$, Yu.V. Gurchin ${ }^{1}$, A.Yu. Isupov ${ }^{1}$, M. Janek ${ }^{2}$, A.N. Khrenov ${ }^{1}$, \\ A.K. Kurilkin ${ }^{1}$, P.K. Kurilkin ${ }^{1}$, V.P. Ladygin ${ }^{1}$, N.B. Ladygina ${ }^{1}$, S.M. Piyadin ${ }^{1}$, \\ S.G. Reznikov ${ }^{1}$ and I.E. Vnukov ${ }^{3}$ \\ ${ }^{1}$ Joint Institute for Nuclear Researches, Joliot-Curie St., 6, Dubna, 141980, Russia \\ 2 Physics Department, University of Zilina, Zilina, Slovakia \\ ${ }^{3}$ Belgorod State University, Pobedy St., 85, Belgorod, 308015, Russia \\ E-mail:aterekhin@jinr.ru
}

\begin{abstract}
The results of study of dp-elastic scattering at the energies from 500 to $1000 \mathrm{MeV} /$ nucleon at Nuclotron JINR are reported. The differential cross section data were obtained for angles range of 70-120 deg. in the c.m.s. The results are compared with existing experimental data.
\end{abstract}

XXII International Baldin Seminar on High Energy Physics Problems

Dubna, Russia

September,15-20 2014

${ }^{*}$ Speaker. 


\section{Contents}

1. Introduction 2

$\begin{array}{ll}\text { 2. Experiment } & 3\end{array}$

3. Data analysis 4

4. The differential cross section 5

$\begin{array}{ll}\text { 5. Conclusion } & 7\end{array}$

\section{Introduction}

The study of $d p$-elastic scattering at various energies at large angles is one of the directions of DSS (Deuteron Spin Structure) project program [1]. This reaction is the longtime subject of the theoretical and experimental investigations. Now the different theoretical models are developed: Faddeev calculations in the momentum space [2] and configuration space [3], and variational calculations based on the solution of the three-particle Schroedinger equation $[4,5,6]$. The momentumspace Faddeev equations for three-nucleon scattering can now be solved with high accuracy for the most modern two- and three- nucleon forces below $200 \mathrm{MeV} / \mathrm{n}$ of the projectile energy [7, 8]. The discrepancy between the theory and experiment is increasing with increasing energy indicating the possibility of relativistic effects. The theoretical calculations using not only $2 \mathrm{~N}$ forces but also different $3 \mathrm{~N}$ forces $[9,10]$ give the best agreement with experimental data.

The experimental material for $d p$-elastic scattering covers the energy range from tens to thousands MeV/n. The precise data were obtained at RIKEN at the energies of 70, 100 and $135 \mathrm{MeV} / \mathrm{n}$ [11] for the angular range of $10^{\circ}<\theta^{*}<180^{\circ}$. The analogous experiment was performed in RCNP at the energy of $250 \mathrm{MeV} / \mathrm{n}$ [12], where the data on the cross section and complete set of proton spin observables were obtained.

The transition to higher energies will allow one to understand the mechanism of manifestation of the fundamental degrees of freedom at distances of the order of the nucleon size. Glauber scattering theory which takes both single and double interactions in this case is a classic approach $[13,14]$. The experimental data for $d p$-elastic scattering at energies from 425 to $1250 \mathrm{MeV} / \mathrm{n}$ are presented in [15]-[25]. The differential cross section data for center-of-mass angles between $91^{\circ}$ and $164^{\circ}$ at energies 470 and $590 \mathrm{MeV} / \mathrm{n}$ were obtained at the National Aeronautics and Administration Space Radiation Effects Laboratory [16]. The data at 580 and $582 \mathrm{MeV} / \mathrm{n}$ were presented in [17] and [18], respectively. The absolute differential cross section was measured at 641.3 and $792.7 \mathrm{MeV} / \mathrm{n}$ in the angular range of $35^{\circ}-115^{\circ}$ and $35^{\circ}-140^{\circ}$, respectively [19]. The data for forward angles at 796 and $1000 \mathrm{Mev} / \mathrm{n}$ were presented in [21] and [22], respectively. The experiment to obtain of the 
data at $1 \mathrm{GeV} / \mathrm{n}$ for the angles of $10^{\circ}<\theta^{*}<170^{\circ}$ at the Brookhaven National Laboratory (BNL) was performed [24]. The new data on the differential cross section of the dp-elastic scattering at $1.25 \mathrm{GeV} / \mathrm{n}$ were obtained with HADES detector [25]. The experimental data are described by the relativistic multiple scattering theory which takes both single and double interactions into account [26].

The experimental setup at Internal Target Station (ITS) [27] at Nuclotron allows to obtain the different observables from $60^{\circ}$ to $140^{\circ}$ in the c.m.s. The measurements of differential cross section were performed at ITS Nuclotron at the energies from 200 to $440 \mathrm{MeV} / \mathrm{n}$ [28]. Here the preliminary data at the energies between 500 and $1000 \mathrm{MeV} / \mathrm{n}$ are presented.

\section{Experiment}

The measurements were performed at ITS [27] at Nuclotron JINR. New ITS DAQ system was used during data taking [29]. The elastically-scattered deuterons and protons were counted by two pairs of detectors placed symmetrically with respect to the beam direction. This allows to improve the quality of the experiment. All deuteron- and proton-counters are based on the Hamamatsu H7416MOD. Another two detectors based on the FEU-85 and FEU-63 were used to count of the quasi-elastically-scattered protons [30]. The layout of the counters with respect to the beam direction for energy $1000 \mathrm{MeV} / \mathrm{n}$ is shown in Fig.1. The $D_{1,2}, P_{1,2}$ and $P P_{1,2}$ are deuteron-,proton- and pp-detectors,respectively. All counters were placed in horizontal plane. The DP-detectors were rotated to give an angular range of the $\theta_{l a b}=19^{\circ}$ to $50^{\circ}\left(\theta_{c . m .}=70^{\circ}\right.$ to $\left.120^{\circ}\right)$. The PP-detectors were mounted at the angle corresponding to quasi-elastic scattering at $\theta_{\text {c.m. }}=90^{\circ}$ and remained stationary throughout the experiment for each energy. The size of the D-,P- and PP-

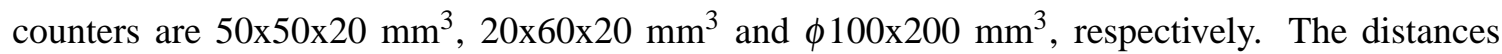
between $P_{1,2^{-}} D_{1,2^{-}}$and $P P_{1,2^{-}}$counters and point the beam interaction with the target are 63, 60 and $100 \mathrm{~cm}$, respectively. The angular spans of $P_{1,2^{-}}, D_{1,2^{-}}$and $P P_{1,2^{-}}$detectors were $2^{\circ}, 5^{\circ}, 10^{\circ}$ in the laboratory system, which corresponds to $4^{\circ}, 10^{\circ}$ and $20^{\circ}$ in the c.m.s., respectively.

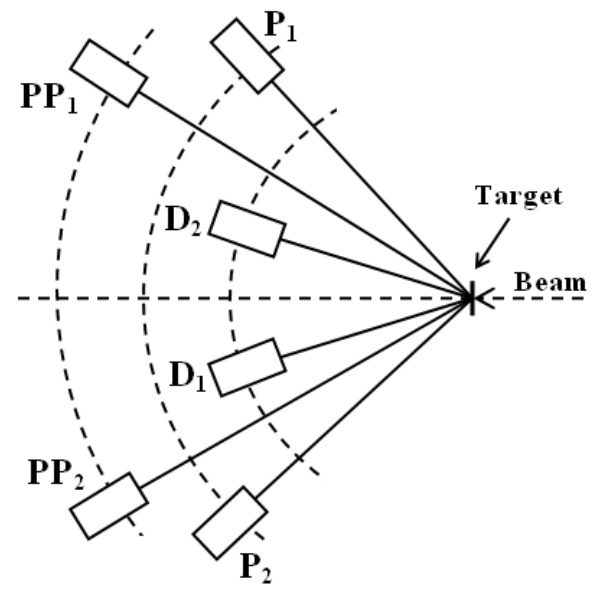

Figure 1: Layout of the counters with respect to the beam direction. $D_{1,2}, P_{1,2^{-}}$deuteron and proton detectors, $P P_{1,2^{-}}$ detectors to registered of the pp-quasi-elastic scattering. 
In the case measurements at lower energies the minor change was made in the scheme of de-

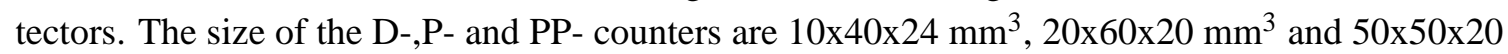
$\mathrm{mm}^{3}$, respectively. All detectors are based on the Hamamatsu H7416MOD. The PP-counters were mounted at the distance $60 \mathrm{~cm}$.

The VME based data acquisition system was used for the data taking from scintillation detectors [31]. TQDC16 module allows to measure the amplitude and time appearance of the signal. Each module is separated on the two parts with 8 input channels having own first level trigger logics. In the current experiment the first level trigger signal was appeared when the signal from one module part coincides with the signal from any channel of other part.

\section{Data analysis}

The data processing at $1000 \mathrm{MeV} / \mathrm{n}$ is shown below as an example. The procedure to obtain differential cross section data begins by analysis of the amplitude spectra. The signal amplitudes correlation for D- and P- detectors was made. Then the graphical cut was imposed to select dpelastic scattering particles (Fig. 2).

The next stage is the application of the temporary gates on the deuteron and proton time difference spectra to estimate the background in the amplitude data. The subtraction of the timing signal from D- and P- counters was made by using the cut for signal amplitudes correlation (Fig 3). In this distribution the dp-elastic scattering events (I domain) and the background (II and III domains) are selected so that the width of both domains are equal.

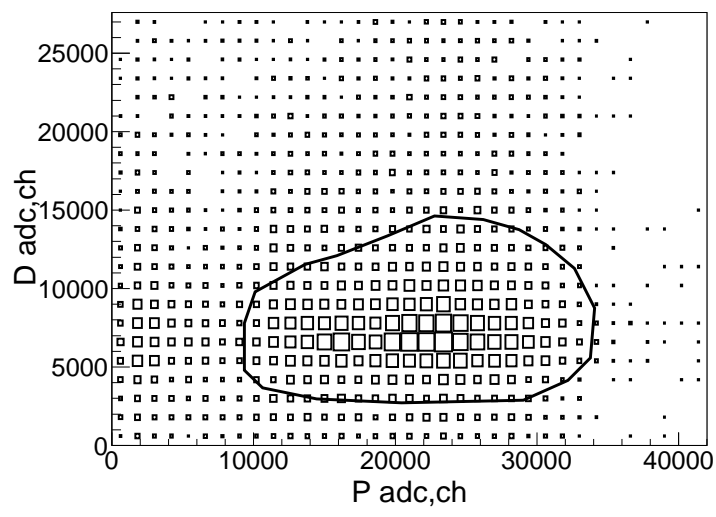

Figure 2: The signal amplitudes correlation for $\mathrm{D}$ and $\mathrm{P}$ detectors at $1000 \mathrm{MeV} / \mathrm{n}$. The solid line is the graphical cut to select dp-elastic scattering events.

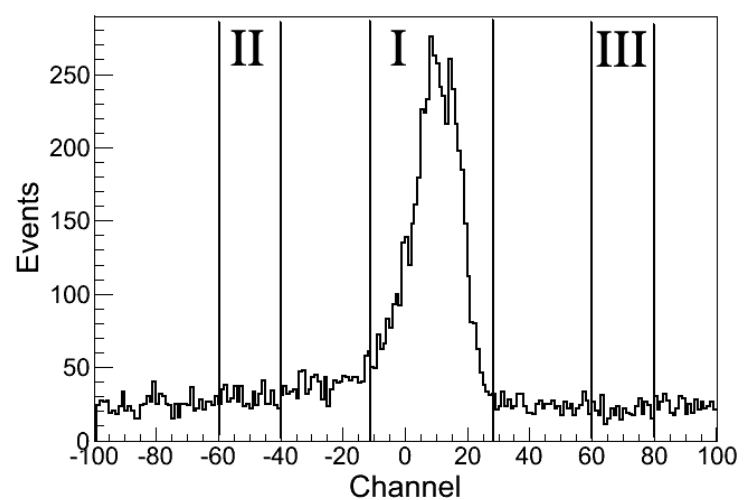

Figure 3: The subtraction of the timing signal from $D$ and P- counters for $\theta^{*}=70^{\circ}$ in the c.m.s. at $1000 \mathrm{MeV} / \mathrm{n}$.

The amplitude distribution for proton counter by using these timing gates is shown in Fig. 4 A. The subtraction of the resulting spectra allows to reduce of the background (Fig $4 \mathrm{~B}$ ).

Analogous procedure was performed for data by using $\mathrm{CH}_{2}$-target as well as C-target.

The next stage is the $\mathrm{CH}_{2}-\mathrm{C}$ subtraction procedure. The carbon background subtraction normalization coefficient $k$ is deduced from the interval $a_{\min }<a<a_{\max }$, where $a$ - channels of $\mathrm{CH}_{2}$ - 

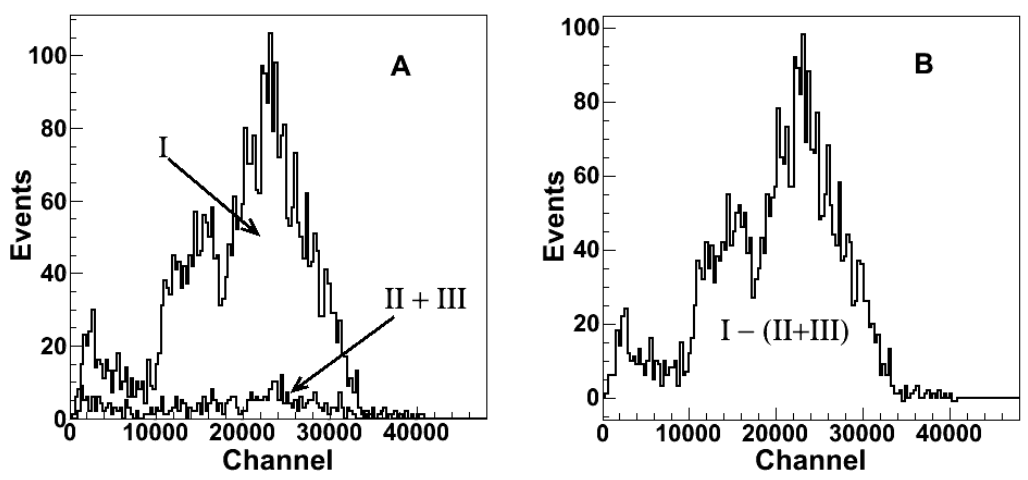

Figure 4: The cleaning procedure of the amplitude spectrum by using of the timing gates.

and C-amplitude distributions:

$$
k=\frac{\left.N_{C H_{2}}\right|_{a_{\min }<a<a_{\max }}}{N_{C} a_{\min }<a<a_{\max }} .
$$

Here $\mathrm{N}_{\mathrm{CH}_{2}}$ and $\mathrm{N}_{\mathrm{C}}-\mathrm{CH}_{2}$ - and $\mathrm{C}$-amplitude distributions integrals in $a$-interval. The carbon background can be subtracted as:

$$
N_{d p}=N_{C H_{2}}-k N_{C}
$$

were $N_{d p}$ - resulting dp-elastic scattering distribution, $\mathrm{N}_{\mathrm{CH}_{2}}$ - total $\mathrm{CH}_{2}$-distribution, $\mathrm{kN}_{\mathrm{C}}$ - normalized $C$-distribution. The subtraction resulting spectra allows to obtain the dp-elastic scattering events.

\section{The differential cross section}

In Fig. 5 the data for differential cross section at $1000 \mathrm{MeV} / \mathrm{n}$ are compared with world data and with the theoretical predictions.

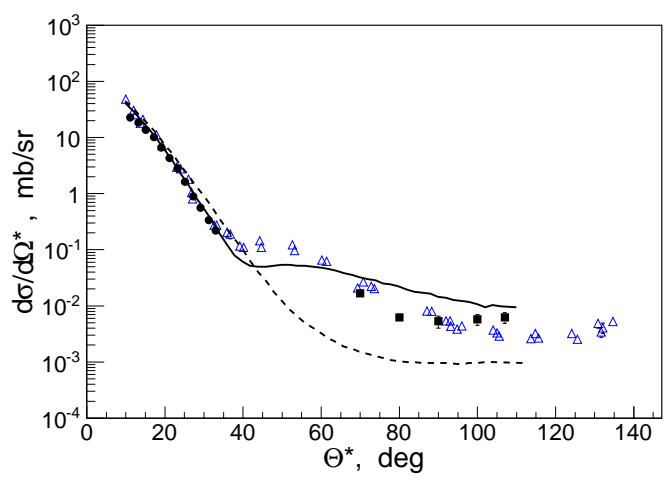

Figure 5: The differential cross section for $d p$-elastic scattering at $1000 \mathrm{MeV} / \mathrm{n}$. Squares - the results of this work, circles - data from [22], triangles - data from [24], line - the theoretical calculations without DS term. 
New data are shown by the solid squares. The errors are the statistical only. The systematic error due to normalization and $\mathrm{CH}_{2}-\mathrm{C}$ subtraction procedure $\approx 30 \%$. The data obtained earlier for forward angles [22] are shown by the solid circles. The open triangles are world data from [24] obtained with a monochromatic protons beam at the Brookhaven Cosmotron by using a liquid-deuterium target. The data at Nuclotron are normalized to data from [24] at the $70^{\circ}$ in the c.m.s. The shape of the angular dependence of new data agrees with the behaviour of the world data. The dashed and solid lines are the calculations without and with double scattering term, respectively. One can see that the single scattering mechanism does not reproduce the experimental data at the scattering angles $\theta^{*}$ larger than $45^{\circ}$. The inclusion of the double scattering term in the calculations provides better agreement with the experimental results. However, some discrepance remains. Probably, taking into account new reaction mechanisms like explicit $\Delta$-isobar excitation will improve the description of the data. The preliminary data for differential cross section at 500,650 and $750 \mathrm{MeV} / \mathrm{n}$ are shown in Fig. 6, Fig 7 and Fig.8, respectively. One can see, the

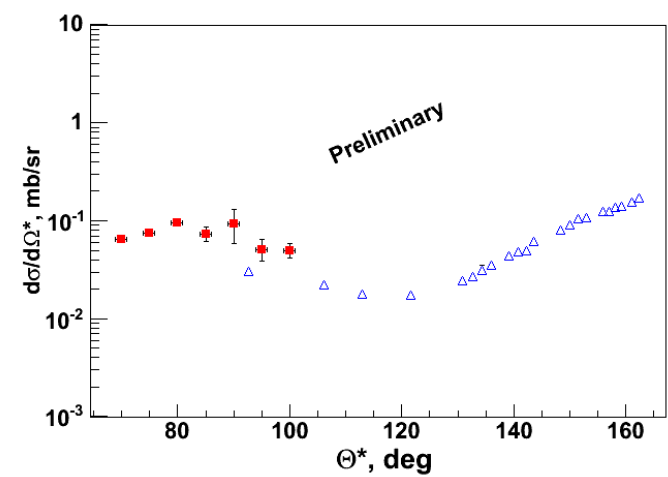

Figure 6: The differential cross section for dp-elastic scattering at $500 \mathrm{MeV} / \mathrm{n}$. Squares - the results of this work, triangles - data from [16].

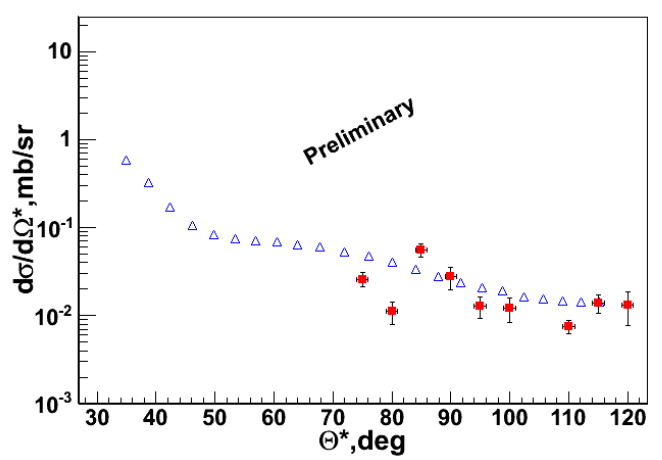

Figure 7: The differential cross section for dp-elastic scattering at $650 \mathrm{MeV} / \mathrm{n}$. Squares - the results of this work, triangles - data from [19].

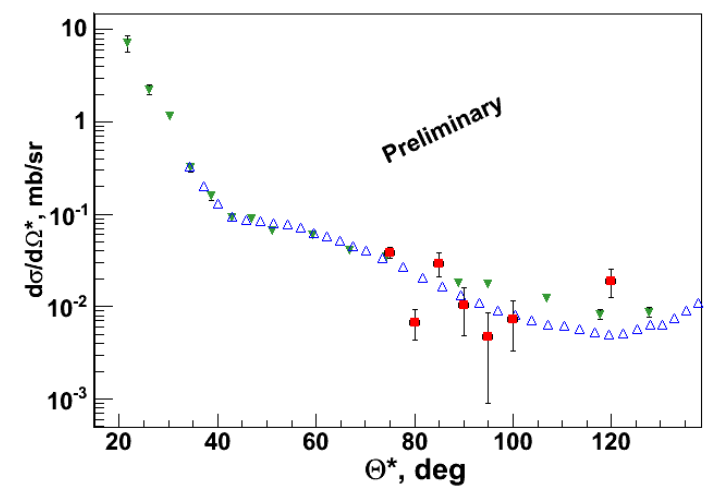

Figure 8: The differential cross section for $d p$-elastic scattering at $750 \mathrm{MeV} / \mathrm{n}$. Squares - the results of this work, open triangles - data from [19], solid triangles - data from [23]. 
shapes of the angular dependence of data obtained at Nuclotron are in the reasonable agreement with the behavior of the world data $[23,24,19,16]$.

\section{Conclusion}

The procedure on the $d p$-elastic scattering differential cross section at high energies at ITS at Nuclotron using $\mathrm{CH}_{2}-\mathrm{C}$ subtraction is established.

The preliminary differential cross section data for $d p$-elastic scattering at 500,650,750 and $1000 \mathrm{MeV} / \mathrm{n}$ are obtained. The results are compared with existing data for similar values of energies.

The data obtained at $1000 \mathrm{MeV} / \mathrm{n}$ are compared with the calculations performed within the framework of the relativistic multiple scattering theory [26]. It is shown that taking into account the double scattering term improves the description of the obtained experimental results.

The work has been supported in part by the RFBR grant 13-02-00101a.

\section{References}

[1] V.P. Ladygin et al., Short-range correlation studies in collisions of polarized nuclei at Nuclotron-M, Eur. Phys. J. Web of Conf. 3 (2010) 04004;

V.P. Ladygin et al., Few-body studies at Nuclotron-JINR, Few Body Syst. 55 (2014) 709.

[2] H. Witala, Th.Cornelius, W.Glockle, Elastic scattering and break-up processes in then-d system, Few-Body Systems 3 (1988) 123.

[3] J.L. Friar, G.L.Payne, W.Glockle, D.Huber, H.Witala, Benchmark solutions for n-d breakup amplitudes, Phys.Rev. C51 (1995) 2356.

[4] A. Kievsky, M.Viviani, S.Rosati, Polarization observables in $\mathrm{p}$ - d scattering below 30-MeV, Phys.Rev. C64 (2001) (024002).

[5] M. Viviani, A.Kievsky, S.Rosati, The Kohn variational principle for elastic proton-deuteron scattering above deuteron breakup threshold, Few-Body Syst. 30 (2001) 39.

[6] A. Deltuva et al., Benchmark calculation for proton-deuteron elastic scattering observables including Coulomb, Phys.Rev. C71 (2005) 064003.

[7] W. Glockle et al., The three-nucleon continuum: achievements, challenges and applications, Phys.Rep. 274 (1996) 107.

[8] J. Kuros-Zolnierczuk, H.Witala et al., Three-Nucleon Force Effects in Nucleon Induced Deuteron Breakup: Comparison to Data (II), Phys.Rev. C66 (2002) 024004.

[9] S.A. Coon, H.K.Han, Revorking the Tucson-Melbourne Three-Nucleon Potential, Few-Body Systems 30 (2001) 131.

[10] B.S. Rudliner et al., Quantum Monte Carlo calculations of nuclei with A $\leq$ 7, Phys. Rev. C56 (1997) 1720.

[11] K. Sekiguchi et al., Complete set of precise deuteron analyzing powers at intermediate energies: Comparison with modern nuclear force predictions, Phys. Rev. C65 (2002) 034003.

[12] K. Hatanaka et al., Cross section and complete set of proton spin observables in pd elastic scattering at 250MeV, Phys.Rev. C66 (2002) 044002. 
[13] V. Franco, R.J.Glauber, Small-Angle High-Energy Scattering by Deuterons, Phys. Rev. Lett. 16 (1966) 944.

[14] V. Franco, E.Coleman, Double Scattering in High-Energy Elastic Collisions with Deuterons, Phys.Rev.Lett. 17 (1966) 827.

[15] N.E. Booth et al., Proton-deuteron elastic scattering at 1.0 GeV/c, Phys. Rev. D4(5) (1971) 1261.

[16] J.C. Alder et al., Elastic pd scattering at 316,364,470 and $590 \mathrm{MeV}$ in the Backward Hemisphere, Phys. Rev. C6(6) (1972) 2010.

[17] J.S. Vincent et al., Large-angle p-d scattering at $580 \mathrm{MeV}$, Phys. Rev. Lett. 24(5) (1970) 236.

[18] E.T. Boschitz et al., Elastic Scattering of 600-MeV Protons from H, D, ${ }^{3} \mathrm{He}$ and $4^{H}$ e, Phys. Rev. C6(2) (1972) 457.

[19] E. Culmez et al., Absolute differential cross section measurements for proton-deuteron elastic scattering at 641.3 and $792.7 \mathrm{MeV}$, Phys. Rev. C43(5) (1991) 2067.

[20] M.G. Albrow et al., Asymmetry in the scattering of protons on polarized deuterons at $1.21 \mathrm{GeV} / \mathrm{c}$, Phys. Lett. B35(3) (1971) 247.

[21] F. Irom et al., Measurements of small elastic p-d scattering at $796 \mathrm{MeV}$ using a recoil method, Phys. Rev. C28(6) (1983) 2380.

[22] A.A. Terekhin et al., Measurement of the differential cross-section and deuteron vector analyzing power in dp-elastic scattering at 2.0-GeV, Eur. Phys. J. A48 (2012) 182.

[23] E. Winkelmann et al., Proton-deuteron elastic scattering at 800 MeV, Phys. Rev. C21 (1980) 2535.

[24] G.W. Bennet et al., Proton-deuteron scattering at 1 BeV, Phys. Rev. Lett. 19 (1967) 387.

[25] P.K. Kurilkin et al., New data on the differential cross section of the dp-elastic scattering at $2.5 \mathrm{GeV}$ obtained with HADES detector, in proceedings of the XXI-st International Baldin Seminar on High Energy Physics Problems: Relativistic Nuclear Physics and Quantum Chromodynamics (ISHEPP 2012) POS Baldin-ISHEPP-XXI 040 (2012).

[26] N.B. Ladygina et al., Differential Cross Section of DP-Elastic Scattering at Intermediate Energies, Eur. Phys. J. A42 (2009) 91.

[27] Malakhov A.I. et al., Potentialities of the internal target station at the Nuclotron. Nucl. Instrum. Methods, Phys. Res. A440 (2000) 320.

[28] Yu.V. Gurchin et al., The differential cross-section on dp-elastic scattering at 400-880 MeV obtained at Nuclotron, Nucl.Phys.Proc.Suppl. 245 (2013) 271;

The cross-section in dp-elastic scattering at the energies of 500-MeV, 700-MeV and 880-MeV obtained at the internal target station of nuclotron, Phys.Part.Nucl.Lett. 10 (2013) 243.

[29] A.Yu. Isupov et al., The Nuclotron internal target control and data acquisition system, Nucl. Instrum. Methods Phys. Res. A698 (2013) 127.

[30] S.M. Piyadin et al., The study of the $d p \longrightarrow$ ppn reaction at 500-MeV of the deuteron energy at ITS nuclotron, Nucl.Phys.B (Proc.Suppl.) 219-220, (2011) 251.

[31] http://afi.jinr.ru 